\title{
Enhanced Lipid Metabolism Induces the Sensitivity of Dormant Cancer Cells to 5-aminolevulinic acid- based Photodynamic Therapy
}

\section{Taku Nakayama ( $\square$ Taku.nakayama@kochi-u.ac.jp )}

Center for Photodynamic Medicine, Kochi Medical School, Kohasu, Oko-cho, Nankoku-shi, Kochi, 7838505, Japan

\section{Tomonori Sano}

Kochi Medical School, Kohasu, Oko-cho, Nankoku-shi, Kochi, 783-8505, Japan

\section{Yoshiki Oshimo}

Kitano Hospital, Tazuke Kofukai Medical Research Institute, Japan

\section{Chiaki Kawada}

Department of Urology, Kochi Medical School, Kohasu, Oko-cho, Nankoku-shi, Kochi, 783-8505, Japan

\section{Moe Kasai}

School of Life Science and Technology, Tokyo Institute of Technology, 4259 Nagatsuta-cho, Midori-ku, Yokohama, Kanagawa, 226-8501, Japan

\section{Shinkuro Yamamoto}

Department of Urology, Kochi Medical School, Kohasu, Oko-cho, Nankoku-shi, Kochi, 783-8505, Japan Hideo Fukuhara

Department of Urology, Kochi Medical School, Kohasu, Oko-cho, Nankoku-shi, Kochi, 783-8505, Japan Keiji Inoue

Department of Urology, Kochi Medical School, Kohasu, Oko-cho, Nankoku-shi, Kochi, 783-8505, Japan

\section{Shun-ichiro Ogura}

School of Life Science and Technology, Tokyo Institute of Technology, 4259 Nagatsuta-cho, Midori-ku, Yokohama, Kanagawa, 226-8501, Japan

\section{Research Article}

Keywords: cancer, metastatic disease, chemotherapeutic drugs, radiation, ALA-PDT

Posted Date: February 1st, 2021

DOI: https://doi.org/10.21203/rs.3.rs-128840/v1

License: (c) (i) This work is licensed under a Creative Commons Attribution 4.0 International License. Read Full License 
Version of Record: A version of this preprint was published at Scientific Reports on March 31st, 2021. See the published version at https://doi.org/10.1038/s41598-021-86886-9. 


\section{Abstract}

Cancer can develop into a recurrent metastatic disease with latency periods of years to decades. Dormant cancer cells, which represent a major cause of recurrent cancer, are relatively insensitive to most chemotherapeutic drugs and radiation. We previously demonstrated that cancer cells exhibited dormancy in a cell density-dependent manner. Dormant cancer cells exhibited increased porphyrin metabolism and sensitivity to 5-aminolevulinic acid-based photodynamic therapy (ALA-PDT). However, the metabolic changes in dormant cancer cells or the factors that enhance porphyrin metabolism have not been fully clarified. In this study, we revealed that lipid metabolism was increased in dormant cancer cells, leading to ALA-PDT sensitivity. We performed microarray analysis in non-dormant and dormant cancer cells and revealed that lipid metabolism was remarkably enhanced in dormant cancer cells. In addition, triacsin C, a potent inhibitor of acyl-CoA synthetases (ACSs), reduced protoporphyrin IX (PpIX) accumulation and decreased ALA-PDT sensitivity. We demonstrated that lipid metabolism including ACS expression was positively associated with PpIX accumulation. This research suggested that the enhancement of lipid metabolism in cancer cells induces PpIX accumulation and ALA-PDT sensitivity.

\section{Introduction}

5-Aminolevulinic acid (ALA) is an amino acid that is known to be a precursor molecule in porphyrin biosynthesis such as heme and chlorophyll. Both heme and chlorophyll are biosynthesized from ALA to function as important cofactors in animal and plant cells [1,2]. For instance, heme is an iron-holding porphyrin which functions as the active site in various enzymes such as catalase, cytochrome P450 and in the electron transport chain complexes in mitochondria. Although ALA almost has no cytotoxicity to human body, porphyrin metabolism is quite different in normal cells and tumor cells after ALA administration. In normal cells, administrated ALA will be synthesized to be protoporphyrin IX (PpIX) and almost all will be inserted iron ions to be heme. However, in tumor cells, previous studies revealed PpIX accumulation as well as heme generation [2-6]. PpIX is a fluorescent substance which has maximum excitation at $405 \mathrm{~nm}$ and emission at $635 \mathrm{~nm}$, respectively. Metal-free porphyrins such as PpIX have the capability of fluorescence and oxidative stress generation by light irradiation [2]. As a result, ALA is clinically used in photodynamic diagnosis (ALA-PDD) and photodynamic therapy (ALA-PDT) in various cancers [7].

ALA-PDD and ALA-PDT are widely used clinically because of their high sensitivity and low invasiveness However, the mechanism of PpIX accumulation after ALA administration is still unclear. We previously investigated porphyrin transporters of PpIX high accumulation and low accumulation bladder cancer specimens. This research identified peptide transporter PEPT1 and ATP-binding cassette transporter $A B C G 2$ are key transporters which regulate intracellular PpIX accumulation [8]. In addition, our other research has revealed that plasma membrane $A B C B 6$ exports intermediate porphyrin molecules across the plasma membrane under hypoxic condition [9]. Another report insisted that ABCB6 expression was critical for PpIX accumulation in cancer cells after ALA administration [10]. Altogether, the results of these 
previous studies suggest that PEPT1, ABCB6, and ABCG2 are critically involved in porphyrin metabolism and regulate PpIX accumulation $[2,6]$.

The latency period for cancer to recur can span years or even decades $[11,12]$. This delay can be explained by cancer dormancy [11]. Dormant cancer cells are relatively insensitive to most chemotherapeutic drugs and radiation. Tumor recurrence can occur when such dormant cancer cells reenter the cell cycle $[2,11,13,14]$. Antonija et al. followed the repopulation dynamics of 150 single lentivirus-marked lineages from 10 human colorectal cancers via serial xenograft passages in mice; as a result, they found evidence of a relatively dormant or slowly proliferating cell population in primary human colorectal cancer cells that retains potent tumor propagation potential, thereby preferentially driving tumor growth after chemotherapy [15].

We previously demonstrated that cancer cells exhibited dormancy in a cell density-dependent manner. The high-density 3D culture model, as a dormant cancer cells model, exhibited strong PpIX accumulation and sensitivity to ALA-PDT. In dormant cancer cells, PEPT1 and ABCB6 were upregulated, and ABCG2 was downregulated. PpIX accumulation and ALA-PDT cytotoxicity were enhanced by G0/G1-phase arrestors in non-dormant cancer cells [2]. In addition, heme levels were also increased in dormant cancer cells that had not been exposed to ALA [16]. These results suggest that porphyrin metabolism was enhanced in those cells. In this study, we conducted microarray analysis of human mRNA in PC-3 prostate cancer cells to reveal metabolic changes caused by cellular dormancy and clarify their effects on sensitivity to ALA-PDT.

\section{Methods}

\section{Biochemicals}

ALA hydrochloride was procured from SBI Pharmaceuticals Co., Ltd. (Tokyo, Japan). We obtained DMEM from Thermo Fisher Scientific (Waltham, MA, USA). Antibiotic antimycotic solution (ABAM) was purchased from Nacalai Tesque (Kyoto, Japan). Fetal bovine serum (FBS) was purchased from Thermo Fisher Scientific. Triacsin C was purchased from Cayman Chemical (Ann Arbor, MI, USA). All reagents used in this research were of the highest purity available.

\section{Cells and cell cultures}

The human prostate cancer cell line PC-3 (provided by Dr. Inoue, Kochi University, Kochi, Japan) was maintained in DMEM supplemented with $10 \%(\mathrm{v} / \mathrm{v})$ FBS and $1 \%(\mathrm{v} / \mathrm{v})$ ABAM. Cells were maintained in a $5 \% \mathrm{CO}_{2}$ atmosphere at $37^{\circ} \mathrm{C}[6]$.

\section{D cell culture}

EZSPHERE ${ }^{\circledR}$ 3D cell culture plates were obtained from AGC Techno Glass Co., Ltd. (Tokyo, Japan) and used to culture cancer spheroids. In total, $5 \times 10^{5}$ (S500) or $1.25 \times 10^{5}$ cells (S125) were seeded with $3 \mathrm{ml}$ 
of medium in each 35-mm dish. After 2 days, $1 \mathrm{ml}$ of old medium was carefully replaced with fresh medium. Four days after seeding, 2300 spheroids were formed in each dish [2].

\section{Analysis of PpIX accumulation}

Intracellular PpIX levels were determined using a confocal microscope or microplate reader. Cells were treated with triacsin $\mathrm{C}$ alone for $48 \mathrm{~h}$, followed by co-culture with triacsin $\mathrm{C}$ and ALA for $24 \mathrm{~h}$. Extracellular PpIX was removed via washing by PBS before analysis. An FV-1000D downright laser-scanning confocal microscope (Olympus, Tokyo, Japan) was used for live-cell microscope imaging. The excitation wavelength was set at $405 \mathrm{~nm}$ for PpIX and $635 \mathrm{~nm}$ for DRAQ5 (BioStatus, Loughborough, United Kingdom). The emission wavelength was set at 560-800 nm for PpIX and 655-755 nm for DRAQ5. Laser illumination was set at 3.0\% power for PpIX and 5.0\% power for DRAQ5. All images were acquired using a $\times 60$ oil immersion lens. The images were analyzed using Olympus Fluoview ver. $4.2 \mathrm{~b}$ software. A Cytation 5 microplate reader (BioTek, Winooski, VT, USA) was used for the live-cell relative quantification of intracellular PpIX content. The excitation wavelength was set at 385-425 nm for PpIX and 330-370 $\mathrm{nm}$ for Hoechst 33342 (Thermo Fisher Scientific). The emission wavelength was set at 615-655 nm for PpIX and 430-470 nm for Hoechst 33342. Fluorescence was measured at 10 points in each well.

\section{Quantitative PCR}

Total cellular RNA was purified using an RNeasy Mini kit (QIAGEN, Hilden, Germany). Further, cDNA was generated via reverse transcription using a SuperScript III First-Strand Synthesis System for RT-PCR (Invitrogen, Thermo Fisher Scientific). Quantitative PCR was performed using the StepOnePlus Real-Time PCR System (Applied Biosystems, Thermo Fisher Scientific) [6]. TaqMan probes were selected for PCR (Ki-67, Hs04260396-g1; MCM7, HS00428518-m1; PEPT1, Hs00953898-m1; ABCB6, HS01039213-m1; ABCG2, Hs01053790-m1; ActB, HS01060665-g1). All procedures were performed according to the manufacturer's instructions.

\section{Microarray}

The quality of purified total cellular RNA was measured at 260 and $280 \mathrm{~nm}$ before microarray analysis. We confirmed that the RNA integrity number of all RNA samples was at least 9.50. The Clariom ${ }^{\mathrm{TM}} \mathrm{S} \mathrm{Assay}$ for humans (Thermo Fisher Scientific) was applied for all samples to analyze human mRNA expression. The raw result data were analyzed using Transcriptome Analysis Console ver. 4.0.1.36. The significance of log fold change (logFC) values for RNAs was evaluated using $t$-tests, and the $p$ values associated with logFC values were adjusted for multiple testing using the false discovery rate (FDR) [17, 18]. Significantly different expression was indicated by FDR-adjusted $p<0.05$.

\section{Light irradiation}

Cells were incubated with $\mathrm{ALA}$ at $37^{\circ} \mathrm{C}$ in an atmosphere of $5 \% \mathrm{CO}_{2}$ for $24 \mathrm{~h}$ and exposed to light-emitting diode (LED) irradiation at an intensity of $14.2 \mathrm{~mW} / \mathrm{cm}^{2}(635 \mathrm{~nm})$ for $5 \mathrm{~min}$ by placing the plate below the LED irradiation unit (Bio Research Center, Nagoya, Japan) as previously described [19]. Cells were further 
incubated in the dark overnight, and their viability was then measured using the MTT cell proliferation assay as previously described [2].

\section{Statistical analysis}

Microsoft Office Excel software was used for statistical analysis and graph preparation. An unpaired twotailed $t$-test was used to test the significance of differences between groups. The data were expressed as the mean $\pm S E$ of at least three independent experiments [6].

\section{Results}

\section{Microarray mRNA analysis overview}

We constructed 2D and 3D cultures with different dormant statuses as described in our previous research [2]. The culture time was 4 days for all conditions. In the $2 \mathrm{D}$ culture before analysis, a density of $1.0 \times 10^{3}$ cells $/ \mathrm{cm}^{2}$ represented $20 \%$ confluency, and a density of $4.2 \times 10^{3}$ cells $/ \mathrm{cm}^{2}$ represented $80 \%$ confluency. The spheroid diameter was $125 \mu \mathrm{m}$ for $\mathrm{S} 125$ and $180 \mu \mathrm{m}$ for S500 (Fig. 1A). The cell number in 35-mm dishes was approximately $4.2 \times 10^{3}$ cells $/ \mathrm{cm}^{2}$ as we previously described [2]. The principal component analysis plot confirmed that the first component had a proportional relationship with cell dormancy. However, the second and third components were unclear (Fig. 1B, C). The heat map images and volcano plots for both the 2D and 3D cultures visualized differentially expressed mRNAs (Fig. 1D-G). All mRNA expression results in microarray are presented in Supplementary Table S1

\section{Pathway analysis according to the dormancy status}

We next analyzed metabolic pathway changes in different cellular dormancy models. Interestingly, lipidrelated pathways were upregulated in dormant cancer cells. For example, the cholesterol metabolism map revealed that dormant cancer cells exhibited greater cholesterol synthesis than non-dormant cancer cells (Fig. 2A). In addition, the lipid metabolism-related genes acyl-CoA synthetase medium chain family member 3 and acyl-CoA synthetase short-chain family member $2[20,21]$ were dramatically upregulated in a dormancy-dependent manner (Fig. 2B, C). Therefore, we conducted further analysis of the relationship between lipid metabolism and porphyrin metabolism after ALA administration.

\section{Triacsin C cytotoxicity in PC-3 cells}

Triacsin $\mathrm{C}$ is a potent inhibitor of acyl-CoA synthetases (ACSs), which act downstream of FASN and convert long-chain fatty acids to acyl-CoA [22]. This reaction is a crucial step in several lipid metabolism pathways, including phospholipid biosynthesis, lipid modification of cellular proteins, and $\beta$-oxidation. In mammals, five ACS isozymes have been identified. Previous reports indicated that ACSs suppress apoptosis and that ACS inhibition by triacsin $\mathrm{C}$ could be a rational strategy for amplifying the antitumor effect of etoposide $[22,23]$. In this study, we selected triacsin $C$ as the lipid metabolism suppressor for further study. Triacsin $\mathrm{C}$ at concentrations of up to $8 \mu \mathrm{M}$ did not induce cytotoxicity in PC-3 cells after 72 
h of exposure (Fig. 3). Thus, we selected triacsin concentrations of 1 and $2 \mu \mathrm{M}$ for further porphyrinrelated analyses.

\section{Porphyrin-related transporter expression changes following triacsin C exposure}

We investigated the expression of transporters involved in porphyrin metabolism. We previously revealed that PEPT1, ABCB6, and ABCG2 expression was remarkably changed in dormant cancer cells, leading to increased porphyrin metabolism [2]. Specifically, PEPT1 and ABCB6 were upregulated in dormant cancer cells, whereas ABCG2 was downregulated. $\mathrm{Ki}-67$ and MCM7, which are cell proliferation markers, were downregulated by triacsin $C$ treatment because of its antitumor activity [24] (Fig. 4). PEPT1 and ABCB6 expression was decreased after $72 \mathrm{~h}$ of triacsin $\mathrm{C}$ treatment, whereas ABCG2 was upregulated. These results were consistent with our previous reports.

\section{Triacsin $\mathrm{C}$ reduced $\mathrm{PpIX}$ accumulation after ALA administration}

Next, we investigated PpIX accumulation after triacsin C and ALA exposure. Confocal microscopy revealed that the fluorescence intensity of PpIX was decreased by triacsin $C$ treatment (Fig. 5A). Live-cell relative quantification analysis also supported the decreased $\mathrm{PpIX}$ content following triacsin $\mathrm{C}$ treatment (Fig. 5B). These results are consistent with the results of transporter expression in Fig. 4.

\section{ALA-PDT cytotoxicity was decreased by triacsin C}

ALA-PDT can lead to cell death via necrosis or apoptosis, and it is a highly effective form of therapy for treating superficial basal cell carcinomas. To investigate the cytotoxicity after photoirradiation with triacsin C, we irradiated cells with red light at an intensity of $14.2 \mathrm{~mW} / \mathrm{cm}^{2}$ for $5 \mathrm{~min}$. Triacsin C increased cell viability in the presence of ALA concentrations exceeding $125 \mu \mathrm{M}$ in a concentration-dependent manner (Fig. 6A). Specifically, $2 \mu \mathrm{M}$ triacsin $\mathrm{C}$ increased cell viability by $30 \%$ in the presence of 250 and $500 \mu \mathrm{M}$ ALA (Fig. 6B, C). These results indicated that the cytotoxicity of ALA-PDT has a positive relationship with lipid metabolism.

\section{Discussion}

In this study, we first demonstrated metabolic differences between dormant and non-dormant cancer cells via microarray analysis. Metabolism pathway analysis revealed specific changes in lipid metabolism in dormant cancer cells. Second, we demonstrated that PpIX accumulation was decreased by exposure to triacsin C, a key inhibitor of lipid metabolism. Moreover, ALA-PDT cytotoxicity was decreased by triacsin C treatment. To the best of our knowledge, this is the first study to demonstrate that lipid metabolism affects PpIX accumulation and ALA-PDT therapeutic efficacy. 
Cancer cells are often exposed to a metabolically challenging environment with scarce availability of oxygen and nutrients. This metabolic stress leads to changes in the balance between the endogenous synthesis and exogenous uptake of fatty acids, which are required by cells for membrane biogenesis, energy production pathways including the TCA cycle in mitochondria, and protein modification $[25,26]$. Alterations in lipid metabolism and consequently lipid composition have important therapeutic implications, as they affect the survival, membrane dynamics, and therapeutic responses of cancer cells [25-27]. Although the regulation of lipid metabolism in cancer remains unclear, a previous study described ACS activity in cancer cells. ACSs convert long-chain fatty acids to acyl-CoA. This reaction is a critical step in several lipid metabolic pathways, including phospholipid biosynthesis, lipid modification of cellular proteins, and $\beta$-oxidation $[23,28]$. ACSs are overexpressed in a variety of cancers [29-36]. Mashima et al. identified an ACS inhibitor as a tumor-selective inducer of apoptosis [22, 23]. In this study, triacsin C did not induce cytotoxicity in PC-3 cells, but it remarkably affected porphyrin metabolism.

Microarray analysis revealed the metabolic changes in dormant cancer cells. The cell cycle is strongly repressed in dormant cancer cells, leading to resistance to drugs that target rapid cell proliferation. Contrarily, heme-related pathways and protein synthesis-related pathways are upregulated. We previously reported that dormant cancer cells accumulate heme at higher levels than non-dormant cancer cells in the absence of ALA exposure [16]. In addition, dormant cancer cells exhibit lower glucose uptake than nondormant cancer cells [2]. Because the enzyme cytochrome c oxidase (complex IV), which is also known as a hemoprotein, plays a key role at the end of the electron transport chain [37,38], we speculated that dormant cancer cells switch their metabolic pathway from highly glycolytic ATP synthesis (i.e., Warburg effect) to oxidative phosphorylation-related ATP synthesis by mitochondria. Many anticancer drugs target Warburg effect-related pathways, which might be one reason for tumor recurrence. Triacsin $\mathrm{C}$ represses the TCA cycle by inhibiting ACSs and mitochondrial activity including oxidative phosphorylation-related ATP synthesis [26]; therefore, PpIX accumulation after ALA treatment may be reduced.

ALA-PDT is one of the most promising forms of photodynamic therapy for clinical cancer treatment. ALAPDT is well suited to the treatment of early-stage malignancies, as it produces a superficial effect that preserves the structure and function of underlying and adjacent tissues. The limitations of its use include its restriction to superficial cancers, a lack of efficacy in hypoxic regions [39], and the need for interstitial optic fibers for nodular lesions [40]. Although clinical studies of dermatological, urological, gastroenterological, and gynecological cancers have been widely performed in several countries [40,41], there are no established predictors of ALA-PDT efficacy. Our previous reports indicated that PEPT1 and $A B C G 2$ are key transporters for intracellular PpIX accumulation [42]. Other reports identified ABCB1 as the key transporter that regulates the effects of PpIX [43]. In this study, we demonstrated that lipid metabolism including ACS expression is positively related to PpIX accumulation. These findings suggest that lipid metabolism in tumors may be predictive of ALA-PDT efficacy. Biopsy is a standard method for confirming cancer cells in tumors [44, 45]. Immunostaining of ACSs or other lipid metabolism-related gene in biopsied specimens could have predictive utility. We plan to conduct further research to investigate this hypothesis. 
The results of this study identified lipid metabolism upregulation as a key factor of the enhancement of PpIX accumulation in dormant cancer cells. Although lipid metabolism is considered a key pathway for cancer proliferation, this is the first report to demonstrate that lipid metabolism induces PpIX accumulation after ALA treatment. Moreover, we will continue to investigate other pathways involved in porphyrin metabolism using microarray analysis. Further study should reveal the mechanism and key regulators of PpIX accumulation in cancer cells.

\section{Declarations}

\section{Acknowledgments}

We would like to thank Mr. Hisato Yamasaki (Thermo Fisher Scientific Co., Ltd.) for the lecture about microarray analysis, and Enago (www.enago.jp) for the English language review.

\section{Author Contributions}

Conceived and designed the experiments: T.N., T.S., Y.O., C.K, K.I., and S.O. Performed the experiments: T.N., T.S., Y.O. and C.K. Analyzed the data: T.N., T.S., Y.O. and C.K. Contributed reagents/materials/analysis tools: M.K., S.Y. and H.F. Wrote the paper: T.N. and S.O.

\section{Funding}

The studies in the authors' laboratories were supported by the Kochi Medical School Hospital President's Discretionary (Grant Number: 51010724, Recipient's Name: Taku Nakayama), and the Grant-in-Aid for Scientific Research (C) (No.20K16415) from the Ministry of Education, Culture, Sports, Science and Technology.

\section{Competing interests}

The authors declare no competing interests.

\section{Data availability}

The datasets generated during and/or analyzed during the current study are available from the corresponding author on reasonable request.

\section{Ethics declarations}


This research does not include direct participation of any human/animals. Hence, ethics committee approval, informed consent to participate/publish is not applicable here.

\section{Consent to participate/Consent to publish}

Not Applicable

\section{References}

1. Nakayama, T. et al. Mitomycin C-induced cell cycle arrest enhances 5-aminolevulinic acid-based photodynamic therapy for bladder cancer. Photodiagnosis Photodyn. Ther. 31, 101893 (2020). doi:10.1016/j.pdpdt.2020.101893

2. Nakayama, T. et al. Dormant cancer cells accumulate high protoporphyrin IX levels and are sensitive to 5-aminolevulinic acid-based photodynamic therapy. Sci. Rep. 6, (2016).

3. Tabata, K., Ogura, S. \& Okura, I. Photodynamic Efficiency of Protoporphyrin IX: Comparison of Endogenous Protoporphyrin IX Induced by 5-Aminolevulinic Acid and Exogenous Porphyrin IX. Photochem. Photobiol. 66, 842-846 (1997).

4. Kennedy, J. C. \& Pottier, R. H. Endogenous protoporphyrin IX, a clinically useful photosensitizer for photodynamic therapy. J. Photochem. Photobiol. B. 14, 275-92 (1992).

5. Krammer, B. \& Plaetzer, K. ALA and its clinical impact, from bench to bedside. Photochem. Photobiol. Sci. 7, 283-289 (2008).

6. Nakayama, T. et al. Mitomycin C-induced cell cycle arrest enhances 5-aminolevulinic acid-based photodynamic therapy for bladder cancer. Photodiagnosis Photodyn. Ther. 101893 (2020). doi:10.1016/j.pdpdt.2020.101893

7. Nakayama, T. et al. Photoirradiation after aminolevulinic acid treatment suppresses cancer cell proliferation through the H0-1/p21 pathway. Photodiagnosis Photodyn. Ther. 28, 10-17 (2019).

8. Hagiya, Y. et al. Expression levels of PEPT1 and ABCG2 play key roles in 5-aminolevulinic acid (ALA)induced tumor-specific protoporphyrin IX (PpIX) accumulation in bladder cancer. Photodiagnosis Photodyn. Ther. 10, 288-295 (2013).

9. Matsumoto, K. et al. Effects of plasma membrane ABCB6 on 5-aminolevulinic acid (ALA)-induced porphyrin accumulation in vitro: Tumor cell response to hypoxia. Photodiagnosis Photodyn. Ther. 12, 45-51 (2015).

10. Zhao, S. G. et al. Increased expression of ABCB6 enhances protoporphyrin ix accumulation and photodynamic effect in human glioma. Ann. Surg. Oncol. 20, 4379-4388 (2013).

11. Aguirre-Ghiso, J. A. Models, mechanisms and clinical evidence for cancer dormancy. Nature Reviews Cancer 7, 834-846 (2007).

12. Chaffer, C. L. \& Weinberg, R. A. A perspective on cancer cell metastasis. Science 331, 1559-1564 (2011). 
13. Wenzel, C. et al. 3D high-content screening for the identification of compounds that target cells in dormant tumor spheroid regions. Exp. Cell Res. 323, 131-143 (2014).

14. Kyle, A. H., Baker, J. H. E. \& Minchinton, A. I. Targeting quiescent tumor cells via oxygen and IGF-I supplementation. Cancer Res. 72, 801-809 (2012).

15. Kreso, A. et al. Variable clonal repopulation dynamics influence chemotherapy response in colorectal cancer. Science. 339, 543-548 (2013).

16. Nakayama, T. et al. ALA-Porphyrin Science. 4, 13-17 (2016).

17. Steinbusch, M. M. F. et al. Expression of RMRP RNA is regulated in chondrocyte hypertrophy and determines chondrogenic differentiation. Sci. Rep. 7, (2017).

18. Peffers, M. J. et al. SnoRNA signatures in cartilage ageing and osteoarthritis. Sci. Rep. 10, (2020).

19. Hagiya, Y. et al. Pivotal roles of peptide transporter PEPT1 and ATP-binding cassette (ABC) transporter ABCG2 in 5-aminolevulinic acid (ALA)-based photocytotoxicity of gastric cancer cells in vitro. Photodiagnosis Photodyn. Ther. 9, 204-214 (2012).

20. Luong, A., Hannah, V. C., Brown, M. S. \& Goldstein, J. L. Molecular Characterization of Human AcetylCoA Synthetase, an Enzyme Regulated by Sterol Regulatory Element-binding Proteins*. Eur PMC. 275, 26458-26466 (2000) doi:10.1074/jbc.M004160200.

21. Fujino, T. et al. Molecular Identification and Characterization of Two Medium-chain Acyl-CoA Synthetases, MACS1 and the Sa Gene Product*. J Biol Chem. 276, 35961-35966 (2001) doi:10.1074/jbc.M106651200.

22. Mashima, T. et al. Acyl-CoA synthetase as a cancer survival factor: Its inhibition enhances the efficacy of etoposide. Cancer Sci. 100, 1556-1562 (2009).

23. Mashima, T., Sato, S., Sugimoto, Y., Tsuruo, T. \& Seimiya, H. Promotion of glioma cell survival by acylCoA synthetase 5 under extracellular acidosis conditions. Oncogene 28, 9-19 (2009).

24. Mashima, T. et al. p53-defective tumors with a functional apoptosome-mediated pathway: A new therapeutic target. J. Natl. Cancer Inst. 97, 765-777 (2005).

25. Munir, R., Lisec, J., Swinnen, J. V. \& Zaidi, N. Lipid metabolism in cancer cells under metabolic stress. Br. J. Cancer 120, 1090-1098 (2019).

26. Liu, Q., Luo, Q., Halim, A. \& Song, G. Targeting lipid metabolism of cancer cells: A promising therapeutic strategy for cancer. Cancer Lett. 401, 39-45 (2017).

27. Santos, C. R. \& Schulze, A. Lipid metabolism in cancer. FEBS J. 279, 2610-2623 (2012).

28. Kim, J. H., Lewin, T. M. \& Coleman, R. A. Expression and characterization of recombinant rat acyl-CoA synthetases 1, 4, and 5: Selective inhibition by triacsin $C$ and thiazolidinediones. J. Biol. Chem. 276, 24667-24673 (2001).

29. Cao, Y., Dave, K. B., Doan, T. P. \& Prescott, S. M. Fatty Acid CoA Ligase 4 Is Up-Regulated in Colon Adenocarcinoma. Cancer Res. 61, 8429 LP - 8434 (2001).

30. Cao, Y., Pearman, A. T., Zimmerman, G. A., Mclntyre, T. M. \& Prescott, S. M. Intracellular unesterified arachidonic acid signals apoptosis. Proc. Natl. Acad. Sci. U. S. A. 97, 11280-11285 (2000). 
31. Yeh, C. S. et al. Fatty acid metabolism pathway play an important role in carcinogenesis of human colorectal cancers by Microarray-Bioinformatics analysis. Cancer Lett. 233, 297-308 (2006).

32. Liang, Y. C. et al. Involvement of fatty acid-CoA ligase 4 in hepatocellular carcinoma growth: Roles of cyclic AMP and p38 mitogen-activated protein kinase. World J. Gastroenterol. 11, 2557-2563 (2005).

33. Gassler, N. et al. Impaired expression of acyl-CoA synthetase 5 in sporadic colorectal adenocarcinomas. J. Pathol. 207, 295-300 (2005).

34. Young, K. S. et al. Regulation of cell growth by fatty acid-CoA ligase 4 in human hepatocellular carcinoma cells. Exp. Mol. Med. 39, 477-482 (2007).

35. Sung, Y. K. et al. Fatty acid-CoA ligase 4 is overexpressed in human hepatocellular carcinoma. Cancer Sci. 94, 421-424 (2003).

36. Yamashita, Y. et al. Fatty acid induced glioma cell growth is mediated by the acyl-CoA synthetase 5 gene located on chromosome 10q25.1-q25.2, a region frequently deleted in malignant gliomas. Oncogene 19, 5919-5925 (2000).

37. Fontanesi, F., Soto, I. C., Horn, D. \& Barrientos, A. Assembly of mitochondrial cytochrome c-oxidase, a complicated and highly regulated cellular process. Am. J. Physiol. - Cell Physiol. 291, 1129-1147 (2006).

38. Steffens, G. C. M., Biewald, R. \& Buse, G. Cytochrome c oxidase is three-copper, two-heme-A protein. Eur. J. Biochem. 164, 295-300 (1987).

39. Matsumoto, K. Effects of plasma membrane ABCB6 on5-aminolevulinic acid (ALA)-inducedporphyrin accumulation in vitro: Tumor cellresponse to hypoxia. Photodiagn. Photodyn. Ther 12, 45-51 (2015).

40. Casas, A. Clinical uses of 5-aminolaevulinic acid in photodynamic treatment and photodetection of cancer: A review. Cancer Lett. 490, 165-173 (2020).

41. Matoba, Y., Banno, K., Kisu, I. \& Aoki, D. Clinical application of photodynamic diagnosis and photodynamic therapy for gynecologic malignant diseases: A review. Photodiagnosis Photodyn. Ther. 24, 52-57 (2018).

42. Hagiya, Y. Pivotal roles of peptide transporter PEPT1 and ATP-binding cassette (ABC) transporter ABCG2 in 5-aminolevulinic acid (ALA)-based photocytotoxicity of gastric cancer cells in vitro. Photodiagn. Photodyn. Ther. 9, 204-214 (2012).

43. Yoshioka, E. et al. Enhancement of cancer-specific protoporphyrin ix fluorescence by targeting oncogenic ras/MEK pathway. Theranostics 8, 2134-2146 (2018).

44. Underwood, J. J. et al. Liquid Biopsy for Cancer: Review and Implications for the Radiologist. Radiology 294, 5-17 (2020).

45. Crowley, E., Di Nicolantonio, F., Loupakis, F. \& Bardelli, A. Liquid biopsy: Monitoring cancer-genetics in the blood. Nat. Rev. Clin. Oncol. 10, 472-484 (2013).

\section{Figures}


a

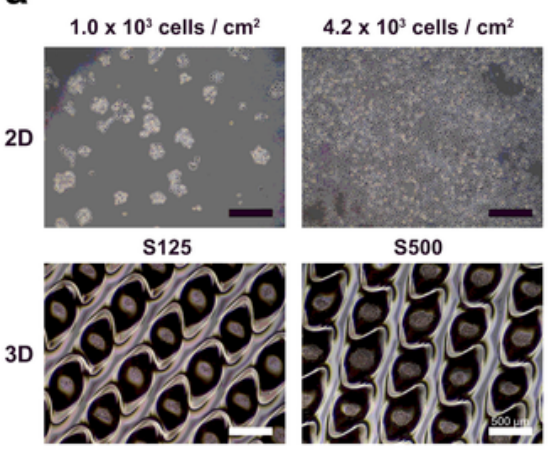

d
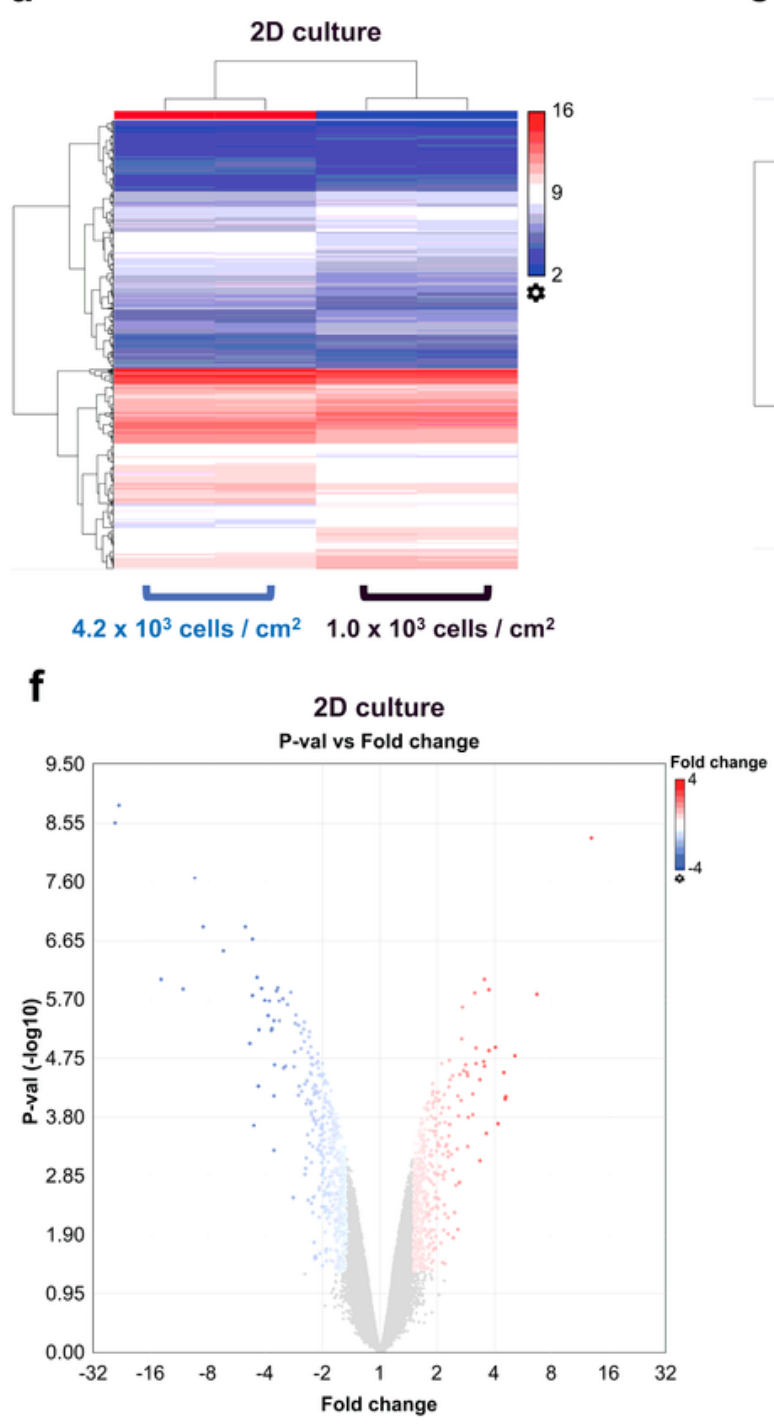

e b

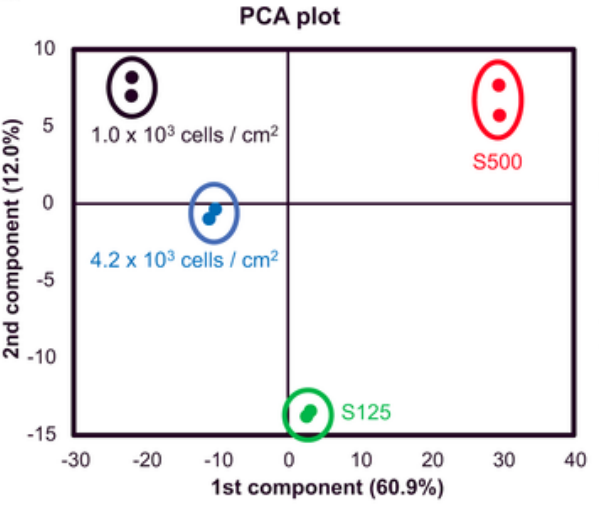

C

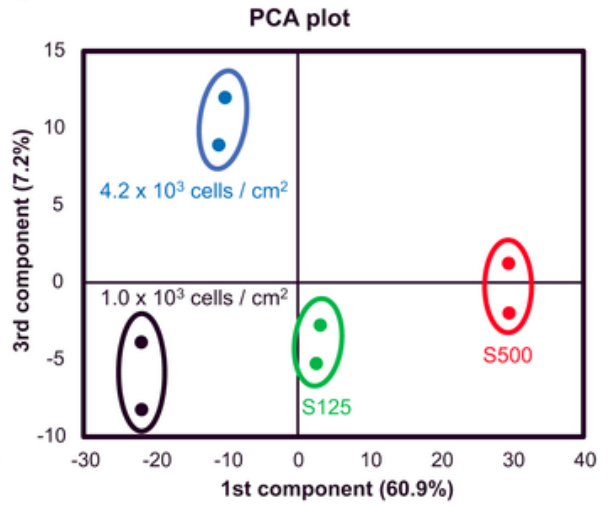

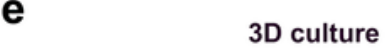
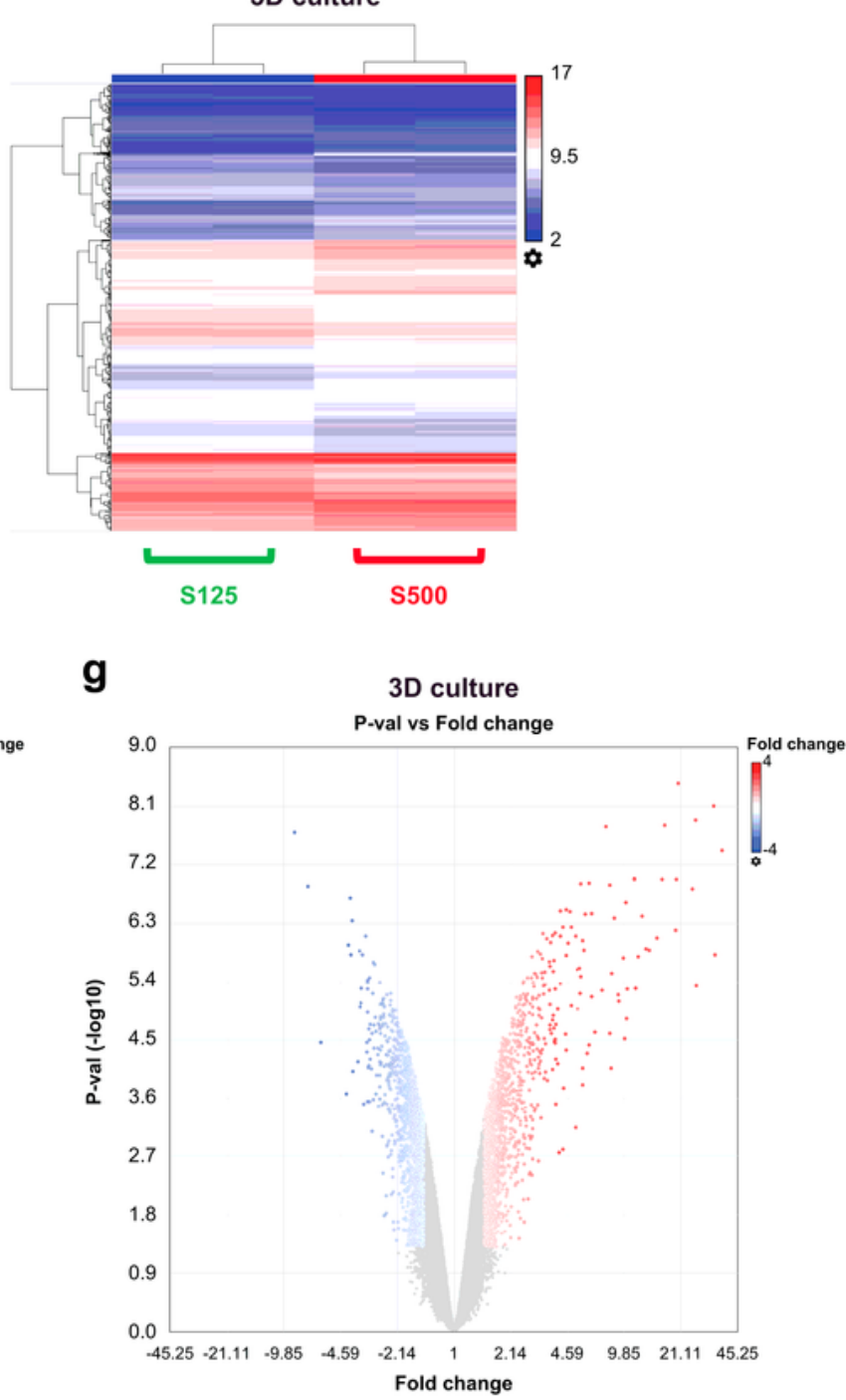

\section{Figure 1}

Microarray profiles of mRNA expression. All data were calculated using Transcriptome Analysis Console ver. 4.0.1.36. (A) Phase contrast images of 2D cultured cells and 3D cultured spheroids. The scale bar is $500 \mu \mathrm{m}$. (B) A 2D principal component analysis (PCA) plot of the first and second components. (C) A 2-D PCA plot of the first and third components. (D-E) 2D and 3D cluster analysis of differentially expressed genes. Red indicates increased expression, and blue denotes decreased expression. (F-G)The volcano 
plot of differentially expressed genes in 2D and 3D culture. Red indicates increased expression, and blue denotes decreased expression.

a

\section{Cholesterol metabolism}

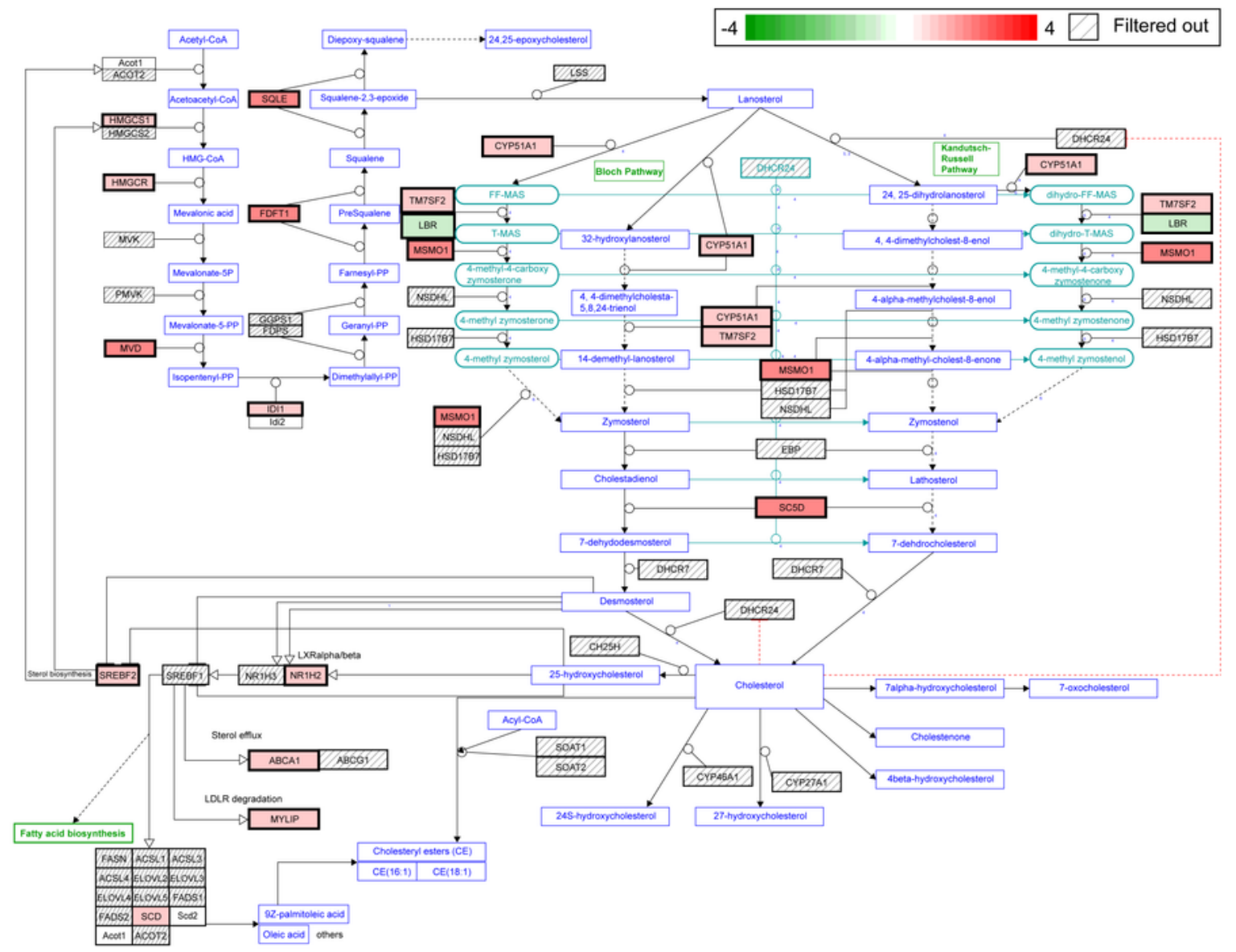

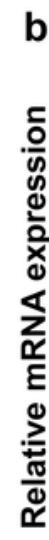

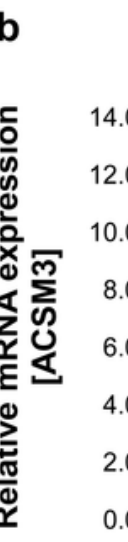

\section{Dormancy}
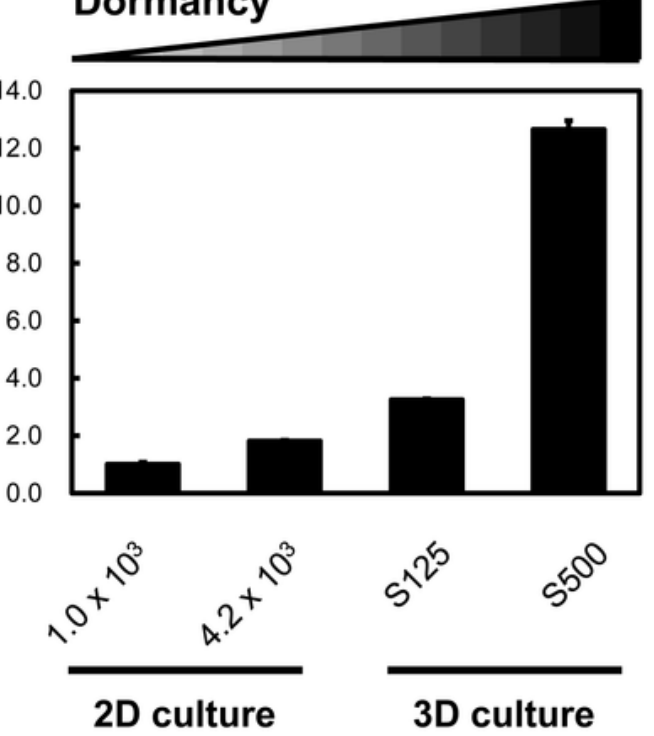
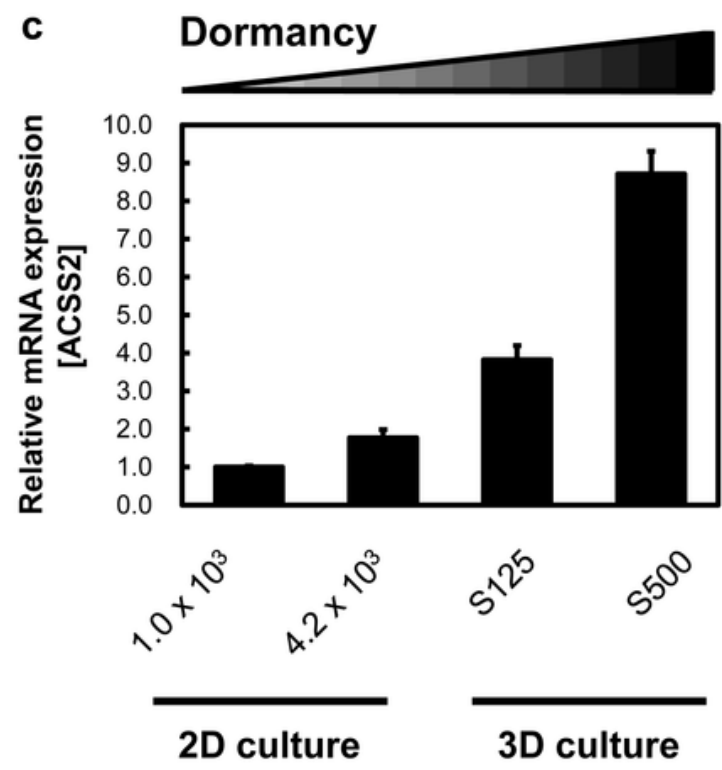

Figure 2

Lipid metabolisms change in dormant cancer cells. (A) Cholesterol metabolism involved in 3D cultured S500 and S125 spheroids. S500 spheroids displayed higher cholesterol metabolism than S125 spheroids. Genes were selected from the "Cholesterol metabolism (includes both Bloch and Kandutsch- 
Russell pathways) (Homo sapiens)" pathway according to Wikipathways. Red indicates increased expression, and green denotes decreased expression. (B) The mRNA expression of acyl-CoA synthetase medium chain family member 3. (C) The mRNA expression of acyl-CoA synthetase short-chain family member 2. All mRNA expression analyses were conducted using Transcriptome Analysis Console ver. 4.0.1.36. Genes with changes in expression exceeding 1.5 -fold and significant at $p<0.05$ genes were extracted. $\mathrm{n}=2$.

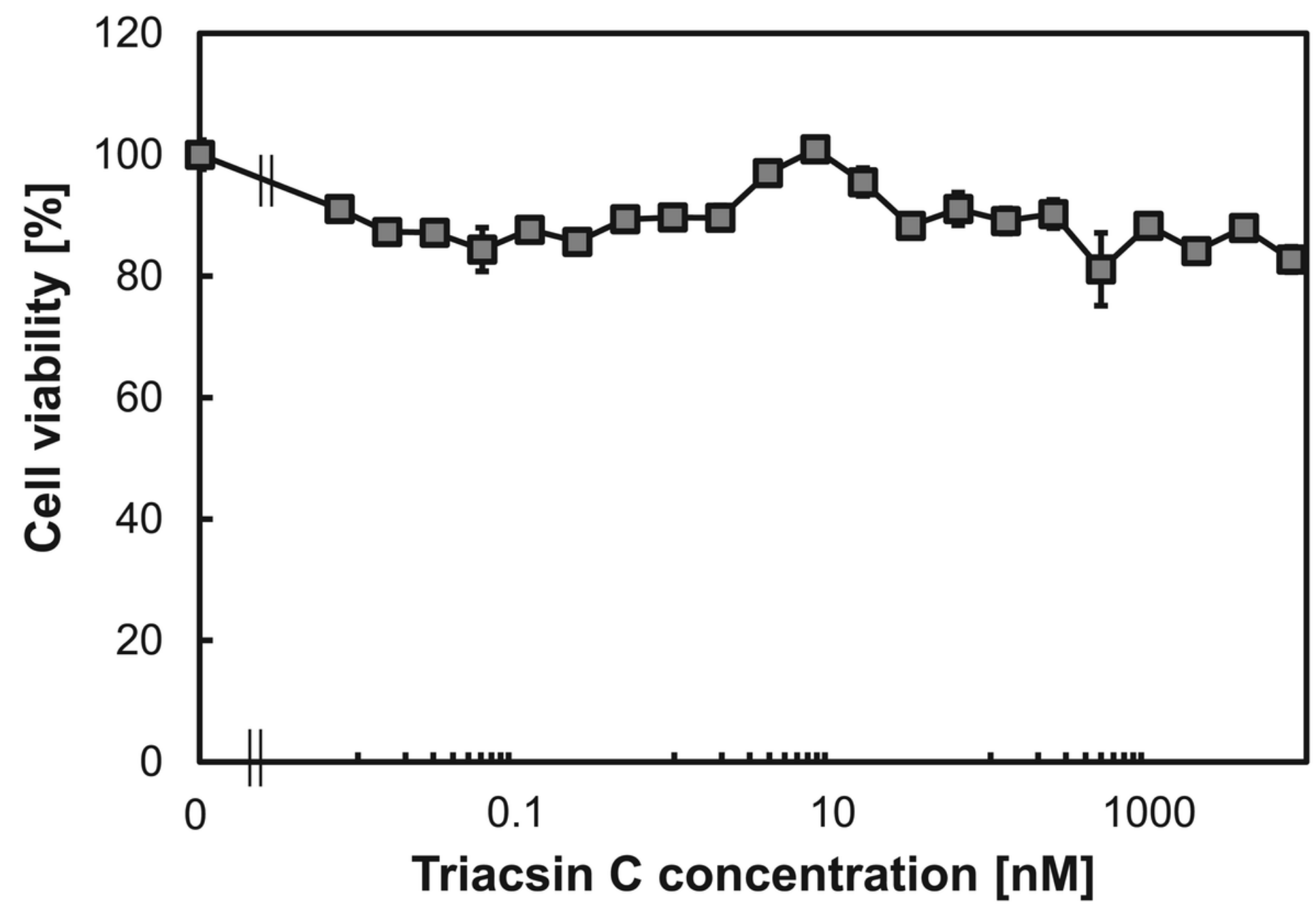

Figure 3

Effects of triacsin C on PC-3 cell proliferation. Cells were treated with various concentrations of triacsin C for $72 \mathrm{~h}$. Cell viability was determined using the MTT assay. $n=4$. Bars represent the SE. 
a
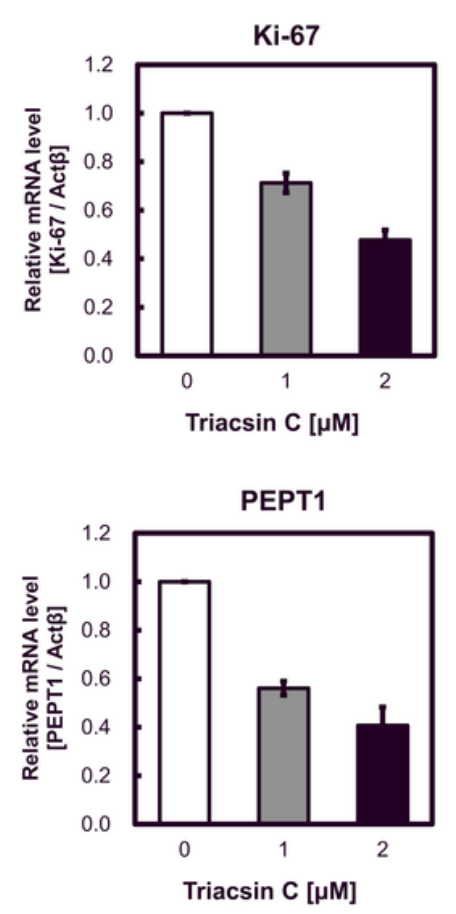
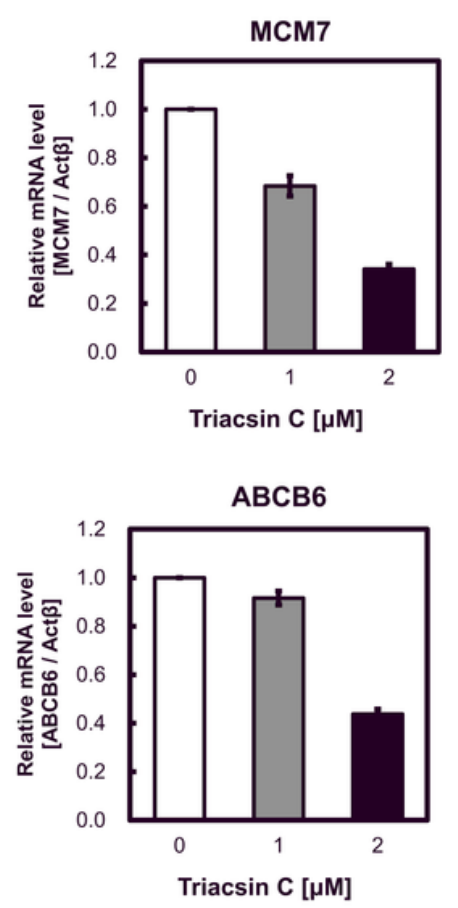

b

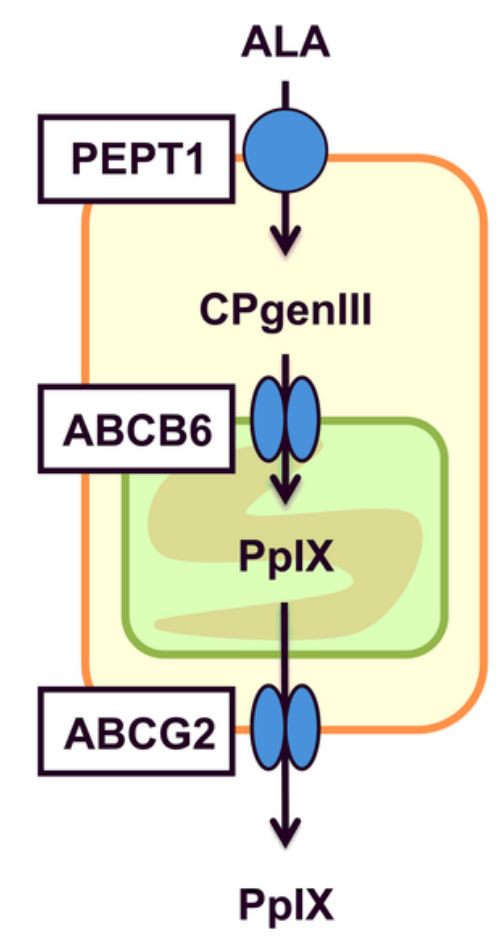

Figure 4

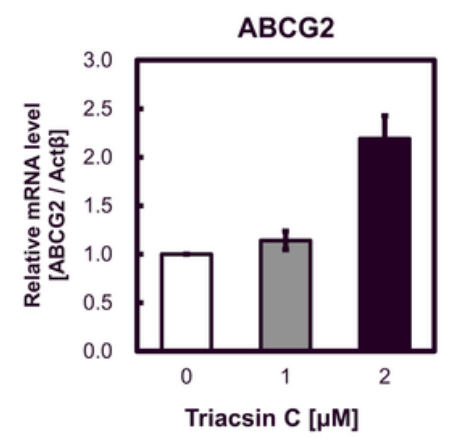

PplX

mRNA expression changes induced by triacsin $C$ treatment. (A) The levels of the cell proliferation markers Ki-67 and MCM7 and porphyrin-related transporters PEPT1, ABCB6, and ABCG2 were measured. The cells were treated with triacsin $\mathrm{C}$ for $72 \mathrm{~h} . \mathrm{n}=3$. Bars represent the $\mathrm{SE}$. (B) Dominant transporters involved in porphyrin metabolism.

a

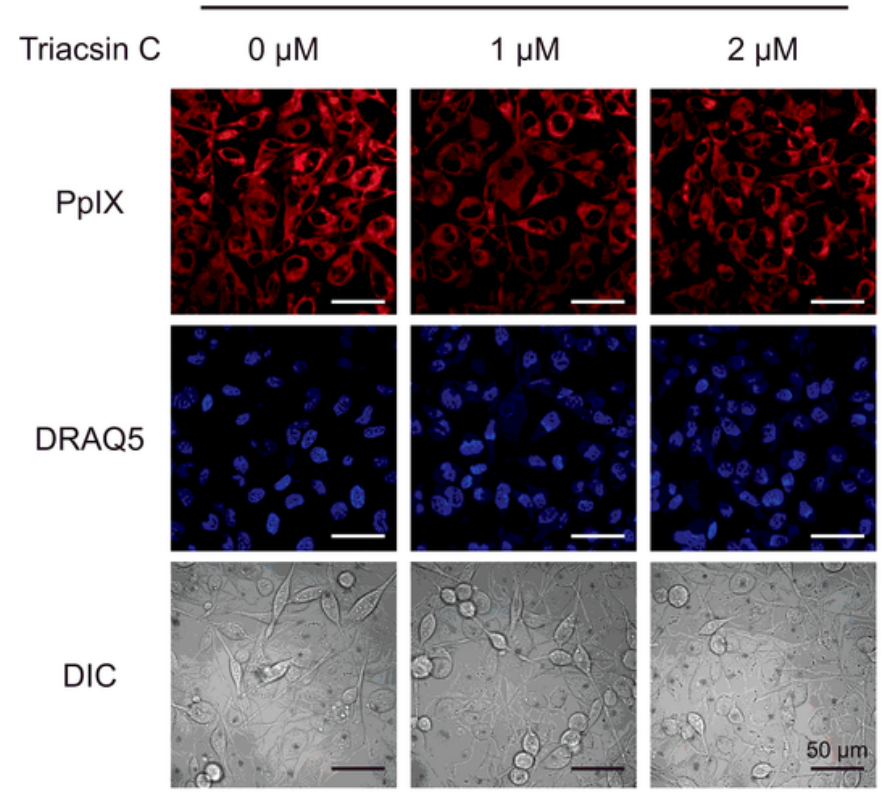

b

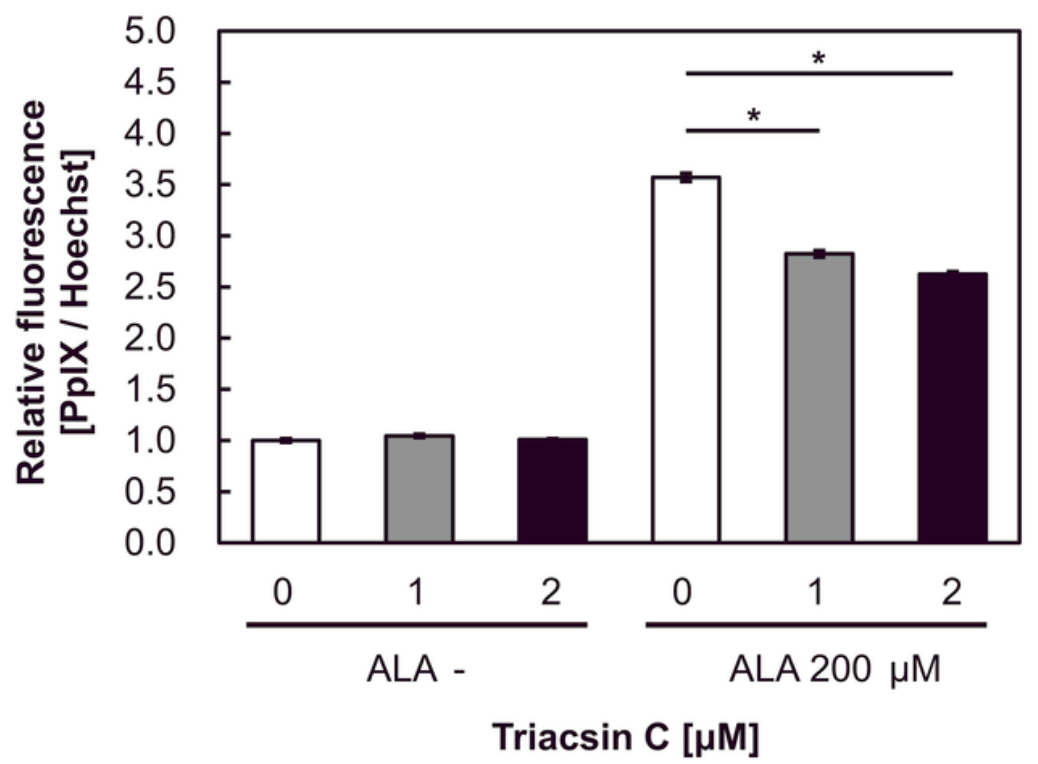

Figure 5 
Triacsin C suppressed protoporphyrin IX (PpIX) accumulation after 5-aminolevulinic acid (ALA) treatment. Cells were treated with triacsin $C$ alone for $48 \mathrm{~h}$ and then co-cultured with triacsin $\mathrm{C}$ and ALA for $24 \mathrm{~h}$. (A) Confocal laser-scanning microscopy images of DRAQ5 (nuclei) and PpIX. Scale bar, $50 \mu \mathrm{m}$. (B) PpIX accumulation in the presence of absence of triacsin $C$ as measured using a microplate reader. $n=3$. ${ }^{*}<$ 0.001 . All bars represent the SE.

a

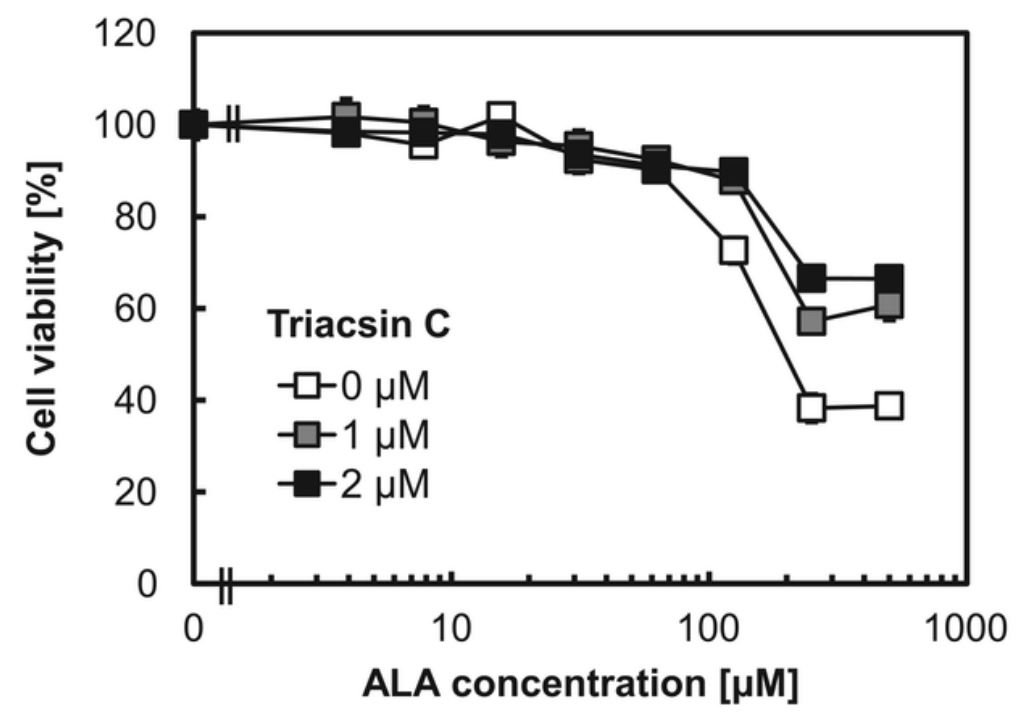

b

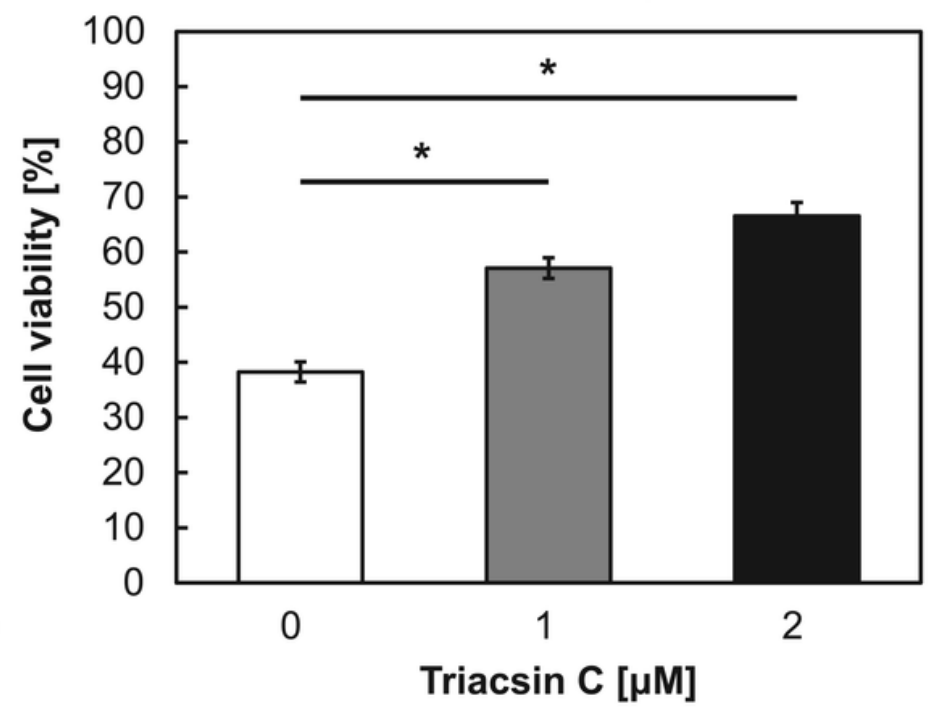

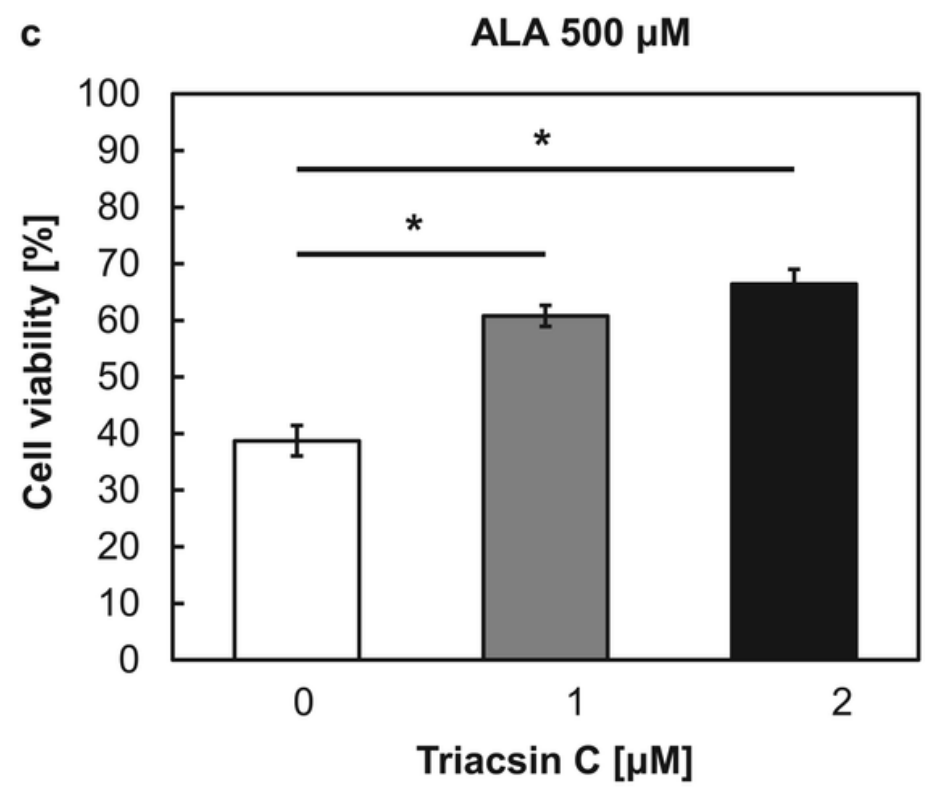

Figure 6

Cell viability after 5-aminolevulinic acid-based photodynamic therapy (ALA-PDT) with or without triacsin C. Cells were treated with triacsin $\mathrm{C}$ alone for $48 \mathrm{~h}$ and then co-cultured with triacsin C and ALA for $24 \mathrm{~h}$. Cells were irradiated with $14.2 \mathrm{~mW} / \mathrm{cm} 2$ light for $5 \mathrm{~min}$. Cell viability was determined on the next day using the MTT assay. (A) Cell viability in the presence of $0-2 \mu \mathrm{M}$ Triacsin $\mathrm{C}$ and various ALA concentrations. (B) Cell viability in the presence of $250 \mu \mathrm{M}$ ALA. (C) Cell viability in the presence of 500 $\mu \mathrm{M}$ ALA. $\mathrm{n}=8 .{ }^{*} \mathrm{p}<0.001$. All bars represent the SE. 


\section{Supplementary Files}

This is a list of supplementary files associated with this preprint. Click to download.

- FC14DECSupportinginformationver.2.docx

- SupplemanteryTableS1.xlsx 